\section{Instantaneity beyond time}

Silvia De Bianchi

More than 2,000 years ago, Aristotle developed a specific concept of temporality connected to change that informed Western culture for centuries.

Today, we are accustomed to associating an imaginary arrow with time or depicting it as a line 'flowing' with a direction. After the advent of quantum mechanics and the formulation of the CPT theorem in $1951,{ }^{1}$ we also agreed to allow this line not to have a priori a unique direction This primitive representation is $s$ intimate to our need to visualise tim that even the most revolutionary thinkers tried to justify it rather than question Even the fathers of quantum mechanics inexhaustibly tried to reconcile the new physical theory with the past. Thei heirs continued using the Hamiltonian, mathematical tool that cannot contrast the mainstream interpretation of flowing time.

\section{General relativity and} beyond

However, the history of physics offers a new radical conception of time embedded in the spacetime concept of genera relativity (GR). In it, we find the roots

for a real change of paradigm in our way of depicting time. First, there is no time describe the system. Second, this theory physic

tells us that what counts is the dynamics of spacetime and matter. We cannot think of one without the other and need new mathematical objects, e.g. tensors, to talk about the universe and describe gravitation

The revolution that GR introduced in physics is well known. However, implications for our conception of time and the investigation of what we call 'atemporality'are still to be fully identified but spacetime as an object. Time is not the advent of quantum computation and associated with an external parameter to applied topology to condensed matter

These relatively new branches of physics are at the beginning of their development. We expect them to bring us into a new technological era that will govern our communication systems. Think of the (a) for instance, which is fundamental in these fields. We We cancot say that the tip hapens in time; there is no duration that can be attributed to this. If a spin-flip occurs, .

The relevance of investigating what we call 'atemporality' is deeply related to the new worldview potentially produced by the advent of quantum computation and applied topology to condensed matter physics.
Instantaneity

Group field theory (GFT) gives another example of instantaneity in current physical theories, according to which there could have been a phase transitio from a non-geometric to a geometric phase of the universe. Again this transition cannot be known or thought of as being in time.

Therefore, another field in which (in 'ill 'n clarification is quantum gravity. With this framework, we find different the methods and aproaches to the che (LC) QG) or GFT. In both cases, thes approaches revindicate the great in thinking of time, but apart from aying that in their theories spacetime emerges, disappears or is not there yet. they are stil unable to provide efinition of atemporality'

\section{The PROTEUS project}

Today we still think of eternity as divided, whereas eternity cannot c. However, under the concept of temporality, Plato inserted another otion besides that of eternity, i.e. instantaneity. The latter is the condition of possibility for thinking of a transition or a sudden switch between opposite out of time. According to Plato, the stant is not at all a part of time, the instant is something that lies in the "in between' and that theoretically justifies transitions that do not have duration and that happen all at once. In other words, in the instant there is no succession but just a switch from $A$ to - $A$ or from $A$ to non-A. In the Platonic picture, we cannot exclude that even temporality is generated from eternity thanks to sudden, instantaneous switch.

This alternative view-neglected for centuries-will be subject to deepe investigation by the PROTEUS research team in the next two years. Scientists, historians and philosophers will try to reflect upon a new definition of instantaneity that will serve as conceptual reservoir for the future theory of quantum gravity quantum information theory and cognates thereby leading to a central role played thereby leading to a central role played by philosophy for future generations.

PROTEUS project partners Avercome the lack of an appropria definition.

In the Platonic dialogues, especially in the Parmenides and the Timaeus, we find the hint for a solution. Before Aristotle reduced the notion of instant to part of time and dominated Western thought with such a postulation for roustion two millent problem of the generation of time and temporalty in a different fashion. He thought that if temporality exists, then its contrary exists as well. Atemporality can be understood, as Aristotle and many Neoplatonic did, as eternity.

The theorem states thatany Lorentz invariant local quantum field theory with a Hermitian e.g. time can

PROJECT SUMMARY

PROTEUS studies the main strategies devised by Western philosophy in epresenting time in cosmology. It aims to modify current metaphysics and it relationship with cosmology in light of recent scientific debates in quantum gravity and quantum cosmology, thereby boosting a new research field in the history and philosophy of cosmology.

PROJECT LEAD

Dr De Bianchi received a PhD in Philosophy at the University of Rome "La Sapienza" (2010) and worked as a post-doc in the UK, Germany, France, and Spain. She is currently a Research Fellow at the University of Milan. In 2017 she received an ERC Starting Grant to investigate different cosmological and quantum gravity models to assess their mplications for our notion of time. From 2021 the projects co-beneficlary institution is the Universitat Autonoma de Barcelona, where the $\mathrm{Pl}$ is leading the activity of seven research team members.

PROJECT PARTNERS

The PROTEUS project is based at the Department of Philosophy of the University of Milan and at the Department of Philosophy of the Autonomous University or Barcelona (UAB). Its members collaborate with the Institute for High Energy Physics
(IFAE). Other collaboration partners include groups from Rome (ILIESI and La Sapienza) and Munich (LMU).

\section{CONTACT DETAILS}

PROTEUS Research Team

Department of Philosophy, Building B,

arcelona 081

욜 +34935868174

www.proteus-pmte.eu

\section{UAB}

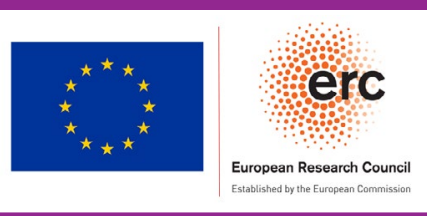

FUNDING

Research Council (ERC) under the European Union's Horizon 2020 research and innovation programme under grant agreement No. 758145 . 